\title{
Clinical outcome of Percutaneous Transforaminal Endoscopic Lumbar Surgery (PTELS) in patient above 60 years of age with lowback pain
}
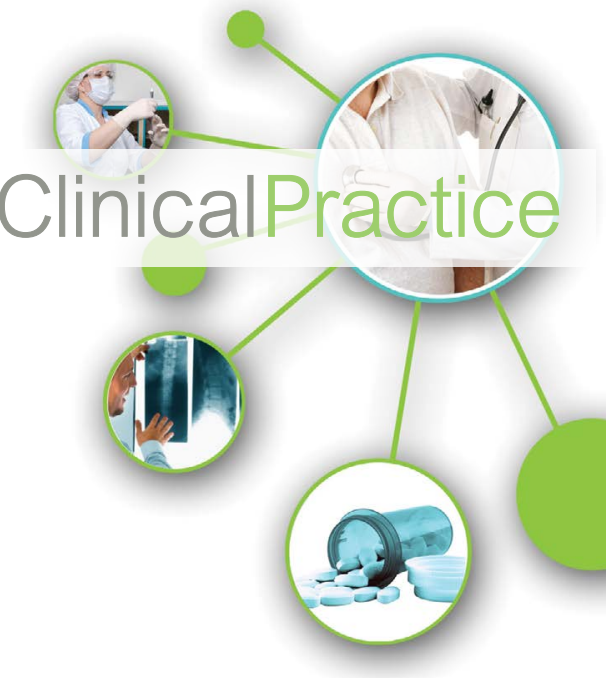

Background: Percutaneous Transforaminal Endoscopic Lumbar Surgery (PTELS) is the latest advance minimal invasive surgical technique in treatment of low back pain.

Objective: To evaluate the outcome of Percutaneous Transforaminal Endoscopic Lumbar Surgeries in patient above 60 years of age and to appraise the efficacy of this technique in treatment of lumbar disc herniation and lumbar spine stenosis in these age group patients.

Materials and Methods: Total 77 patients with age more than 60 years were retrospectively studied, among which 45 patients presented with Lumbar Disc Herniation (LDH) and 22 patients with Lumbar Spinal Stenosis (LSS) who had undergone Percutaneous Transforaminal Endoscopic Lumbar surgery (PTELS) in Zhongda Hospital Affiliated Southeast University from June 2013 to June 2016 A.D. The calculated mean follow up was 20.87 months.

Results: Mean age of operated patient was 68.33 years. Average time of surgery was $87.31+24.746$ minutes. And average hospital stay after surgery was $4.79+2.711$ days. The preoperative and postoperative Oswestry Disability Index (ODI) was $(52.8022+11.98299,16.3513+12.97398, p=0.000)$ indicating statistical significance. The Visual Analog Scale (VAS) of leg and back was $(6.27+1.213,4.79+1.162)$ and $(1.40+1.688,1.30+1.436) p=0.00, p=0.00$ respectively with significant difference before and after surgery. On the basis of MacNab criteria $76.1 \%$ of patients showed excellent or good, $10 \%$ showed fair and $6 \%$ with poor outcome. Overall complication was $10.5 \%$ out of which $3 \%$ (2 cases) were of incomplete removal of content and $7.5 \%$ ( 5 cases) were of reherniation.

In Lumbar Disc Herniation (LDH) there were total 45 patients, of which 26 males and 19 females. In Lumbar Spinal Stenosis (LSS) there were total 22 patients, of which 8 males and 14 females. Time of operation in two group were 87.67+25.486 and $86.59+23.724$ minute respectively with $p=0.537$ which showed no significance difference between two groups. Hospital stay after surgery was $5.09+3.088$ and $4.18+1.593$ days respectively with $p=0.484$. There was no statistically clinical significance difference found between LDH and LSS on the basis of Oswestry Disability Index (ODI) $p=0.863,9$ Visual Analog Scale (VAS) leg and back $p=0.193, p=0.897$, MacNab criteria $p=0.090$.

Conclusion: Percutaneous Transforaminal Endoscopic Lumbar Surgery can be a good alternative technique in spine surgery to relieve symptoms for low back pain with satisfactory outcome, short hospital stay and advantage regarding no need of general anesthesia for elder patient. There is no significant difference between the clinical outcome of Percutaneous Transforaminal Endoscopic Lumbar Surgery (PTES) between lumber disc herniation and lumber spine stenosis of patient above 60 years of age.

KEYWORDS: Percutaneous Transforaminal Endoscopic Lumbar Surgery (PTELS), Percutaneous Endoscopic Lumbar Surgery (PELS), Lumbar Spinal Stenosis (LSS), Lumbar Disc Herniation (LDH)

\section{Introduction}

Low Back pain is one of the common reasons for hospital visit [1-3]. Low back pain is a common disorder involving the muscles, nerves, and bones of the Back [4-6]. Approximately 9 to $12 \%$ of people have LBP at any given point in time and nearly $25 \%$ have reported it at some point over any one-month period [7]. About 70-
$80 \%$ of population experiences low back pain with or without leg pain in lifetime and is one of cause for huge economic burden to family and society [8]. Men and Women are equally affected [9]. LBP is more common among people aged 40-80years [10-12]. Not all patients with back and leg pain need operative treatment but when sign and symptoms progressively increase
Singh Ratish, Hirachan Mangal $P$, Gao Zeng Xin* \& Lin Yu Cheng

Department of spine surgery, Zhongda Hospital affiliated to South East University, Nanjing, China

*Author for correspondence: gaozengxin@outlook.com 
despite of conservative treatment for more than 6 week then the case is considered for surgery [13]. Currently, there are numerous surgical interventions for spinal surgeries, broadly classified as posterior open decompression and percutaneous techniques $[14,15]$. In last 34 years after introduction of percutaneous technique for spine surgery, it has been practiced more and more in every part of the world due to its comparable significant outcome, minimal invasive, shorter time for hospital stay and no need of general anesthesia [16-18]. Our aim of this study was also to evaluate the outcome of percutaneous endoscopic lumbar surgery in elder patients above 60 years of age and also to see the efficacy of this technique in lumbar disc herniation and lumbar spine stenosis in these age group patients.

\section{Material and method}

We retrospectively follow up 77 patients of age above 60 years with Lumbar Disc herniation (LDH) and lumbar spinal stenosis (LSS) that had undergone Percutaneous Transforaminal Endoscopic surgery (PTES) in our hospital from June 2013 to June 2016. Selection of patient for PTES was done by: 1.failure to conservative treatment under medication and physical therapy for more than 6 weeks; 2.Symptoms and physical examination; and 3.Radiological evaluation. Out of 77 patients 6 patients had normal death and 4 patients did not follow up. So, remaining 67 patients were suitable for the study. Follow up was done by telephone inquiry and at outpatient department (OPD) visit. In 67 patients, there were 45 cases of lumber disc herniation and 22 cases of lumber spinal stenosis. On the basis of clinical sign and symptoms, computed tomography (CT), magnetic resonance imaging (MRI), patients were diagnosed as lumber disc hernia and lumber spine stenosis [19].

Self-evaluation questionnaires of the Oswestry disable index (ODI) and MacNab criteria were used for clinical outcomes [20]. Visual Analog Scale (VAS) was used for pain [21]. The evaluation of recurrence of symptoms, complications, duration of hospitalization and length of operative time were reviewed during Outpatient department (OPD) visit and through telephone inquiries. Magnetic resonance imaging (MRI), computed tomography (CT) scans and $\mathrm{x}$-ray were performed in all cases.

IBM SPSS version 24.0 was used to perform statistical analysis. Paired t-test was used to see preoperative and postoperative ODI for all cases. Comparison of LDH and LSS was done using independent sample T-Test, Nonparametric 2 independent sample test, and fisher exact test. A $p<0.05$ was considered statistically significant.

\section{Surgical technique for PTELS}

After all preoperative evaluation, Patient was prepared for surgery. Patient was placed on Operation theatre (OT) table in prone or lateral decubitus position. If patient was placed in prone position then hip and knee were flexed and pillow was placed between lower abdominal and chest so that abdomen would be hanged freely (FIGURE 1A). For lateral position pillow was placed under the waist, which would open up the foramen and allow the Dura to fall down to the contra-lateral side avoiding damage on introduction of the cannula and also reduced intra-abdominal pressure and decrease bleeding. Then under the guidance of c-arm entry point for surgery was marked $8-16 \mathrm{~cm}$ from the mid of vertebra to lateral according to the disc interval that needed to be remove at the angle of 15-25 degree in horizontal plan [22,23]. Under all aseptic condition and local anesthesia (1\%lidocane), identifying anteroposterior (AP) and lateral view with c-arm, a $25 \mathrm{~cm} 18$ gauge needle was placed in the level of herniated disc through the posterolateral approach (FIGURE 1B).

Then an intraoperative disco gram with a 2 $\mathrm{mL}$ mixture of contrast medium, methylene blue at the ratio of 9:1 was performed (FIGURE 1C). If the dye leaked in the epidural space, it indicated tear in annulus [24]. Then guide wire was inserted and the needle was removed. Further, a small skin incision of $8 \mathrm{~mm}$ was made over the guide. A conical dilating rod of 2 $\mathrm{mm}$ was introduced over the guiding wire, and then sequentially the second and third sleeves dilating the soft tissues up to $6.5 \mathrm{~mm}$. Then the reamer was introduced anti clockwise, to avoid damage to the spinal muscles. Continuously checking with the image intensifier, the lamina was reamed and the reamer was advanced safely to 1 or $2 \mathrm{~mm}$ inside the medial pedicular line from smaller to larger reamer (FIGURE 1D). The working cannula was then introduced over the third conical rod. Its tip should be located on the herniated disc. Then image intensifier was removed and working endoscope set was introduced to remove the content in canal under direct visualization (FIGURE 1E). After complete decompression, the Dural sac and the lumber exiting nerve root should be checked 

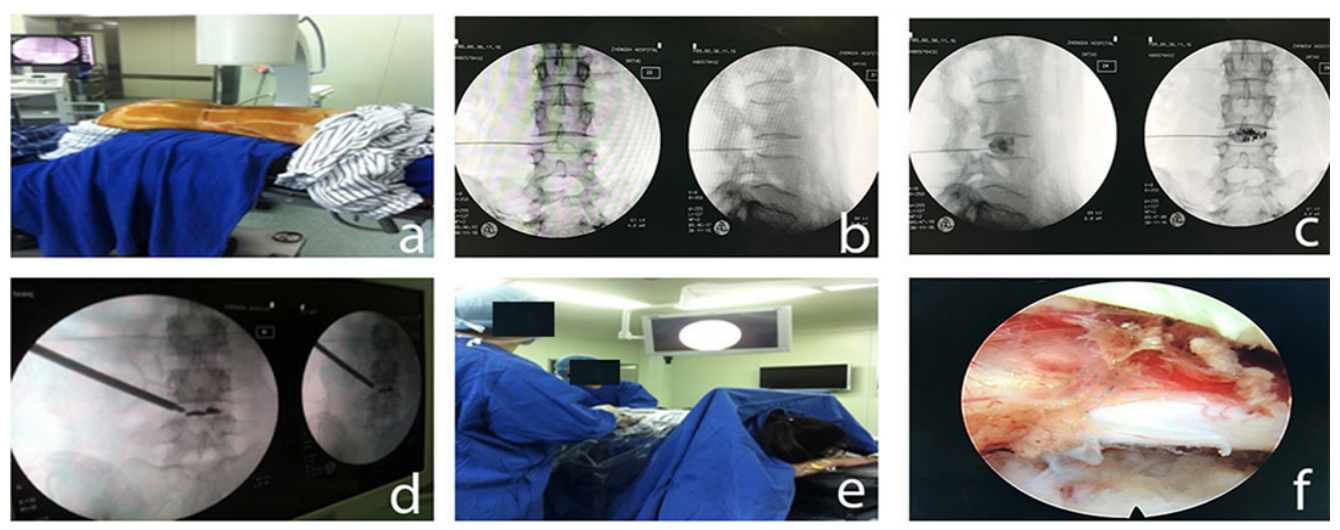

FIGURE 1. Showing operating images during Percutaneous Transforaminal Endoscopic Lumbar Surgery. (A) Positioning of patient during surgery (B) Insertion of needle (C) Discogram with mixture of contrast medium and methylene blue (D) Reaming over dilator (E) Removal of content under direct vision with endoscope (F) Free nerve root after discectomy (18).

for freely movable with the valsalva maneuver (FIGURE 1F). Bleeding of the small vessels was controlled with a flexible bipolar radio frequency probe. Communication with patients was maintained throughout the procedure.

\section{Result}

\section{- Clinical outcomes from PTES of patients above 60 years old}

The total number of patients was 67 out of which 34 male and 33 female. Mean age of operated patient was 68.33 years. Among 67 patients, 45 patients were Lumber disc Herniation and 22 were lumber spine stenosis. Mean follow up was 20.87 months. The average time of operation was $87.31+24.746$ minutes and average hospital stay after operation was $4.79+2.711$ days. The pre and postoperative ODI was $(52.8022+11.98299$, $16.3513+12.97398, \mathrm{p}=0.000)$ with indicating statistically significance. The VAS of leg and back was $(6.27+1.213,4.79+1.162$ and $1.40+1.688$, $1.30+1.436) \mathrm{p}=0.00, \mathrm{p}=0.00$ respectively with significant difference before and after surgery. $76.1 \%$ of patient shows excellent or good, 10 fair and 6 poor out come on basis of $\mathrm{MacNab}$ criteria. There were $3 \%$ ( 2 case) of incomplete removal of content and $7.5 \%$ (5 cases) of reherniation.

\section{- Comparative study of Clinical outcomes of PTES between lumber disc herniation and lumber spine stenosis}

Clinical outcome of PTES between lumber disc herniation and lumber spine stenosis was compared on the basis of ODI, VAS leg and back, MacNab criteria, time of surgery, hospital stay after surgery and complications. In LDH there were total 45 patients, 26 male and 19 female whereas In LSS there was total 22 patients, 8 male and 14 female. The mean age in $\mathrm{LDH}$ and LSS were $66.84+6.098$ years and $71.36+9.302$ years respectively. Time of operation in two groups were $87.67+25.486$ and $86.59+23.724$ minutes respectively with $\mathrm{p}=0.537$ which showed no significance difference between two groups. Hospital stay after surgery was $5.09+3.088$ and $4.18+1.593$ days respectively with $\mathrm{p}=0.484$. There was no statistically clinical significance difference between LDH and LSS on basis of ODI $p=0.863$. For VAS leg and back $\mathrm{p}=0.193, \mathrm{p}=0.897$ which indicate no significant difference between two groups. In case MacNab criteria no statistically significant difference with $\mathrm{p}$ value 0.090 .4 cases $(8.9 \%)$ of re-herniation were seen in $\mathrm{LDH}$ out of 45 and 1 case (4.5\%) of re-herniation and 2 cases $(9.1 \%)$ of incomplete removal of content out of 22 cases were seen in LSS. On the basis of MacNab criteria, LDH group $48.9 \%$ excellent, $28.9 \%$ good, $15.6 \%$ fair and $6.7 \%$ poor were seen. And in LSS $22.2 \%$ excellent, $50.0 \%$ good, 13.6 fair and $13.6 \%$ poor were seen.

\section{Discussion}

Percutaneous Endoscopic surgery is one of the latest emerging techniques in treatment of lumber disc herniation [25]. After the introduction of non-visualized percutaneous central nucleotomy via posterolateral approach by Kambin and Gellman in 1973 and Hijikatain in 1975 and introduction of direct visualization of intervertebral disc space with modified arthroscope by Forest and Housman in 1983 thereafter it has been widely practiced technique for spinal surgery [26-28]. Since then there has 
been many modification in instrument and technique. Recently, there are two techniques widely used for percutaneous endoscopic surgery: 1) Transforaminal approach described by Yeung (Yeung endoscopic spine system technique, YESS) in 1997 and Hoogland (Transforaminal Endoscopic Surgery System, TESSYS) in 1994 [29,30]; 2) Interlaminar approach described by Ruetten in 2007 [31].

After the development of day care surgery concept, minimal invasive technique and idea of no need of general anesthesia, patients are more and more attracted to PELS [32]. On the basis of ODI, VAS, MacNab criteria, and days of hospital stay after surgery, PELS has shown good clinical outcome compared to traditional Open Lumbar discectomy, Micro discectomy and other techniques.

A MacNab criterion is evaluated in four ratings as per their symptomatic relief, excellent, good, fair and poor (Excellent: No pain, No restriction of mobility, Return to normal work and Level of activity; Good: Occasional non radicular pain, Relief of presenting symptoms, Able to return to modified work; Fair: Some improved functional capacity, Still handicapped and/or unemployed; Poor: Continued objective symptoms of root involvement, Additional operative intervention needed at the index level irrespective of the length of postoperative followup) $[33,34]$. Based on the modified MacNab criteria, study done in Taiwan showed $89 \%$ of patients achieved excellent $(28 \%)$ or good (61\%) outcomes after surgery [35]. Different articles till date shows $80-96.7 \%$ good outcome after PTES with MacNab criteria [31,36,37]. Li ZZ et. al. reported case series of 85 patients of lumbar lateral recess stenosis with/without combined HDs treated with PLF-PELD 90.6\% of cases were given "excellent" or "good" of MacNab score [38]. Study to compare PTED with MED has shown no significant difference with $\mathrm{p}=0.99$ (TABLE 1 ).

In our study $76.1 \%$ has excellent or good outcome on basis of MacNab criteria. Our result seems little lower than other studies. We assumed that difference in this value might be due to old age, few sample and other comorbid condition of patients. And all the result of previous study are done in young adult not in these age group. In comparison between LHD and LSS in these age group shows no significant difference with $\mathrm{p}=0.09$ (TABLE 2).

In this study the pre and postoperative ODI was improved from $52.8022+11.98299$ to $16.3513+12.97398$ with statistically significance $\mathrm{p}=0.000$ (TABLE 3) which are similar to the other different studies done till now.

There was no significant difference between LDH and LSS in compare to ODI $\mathrm{p}=0.863$ (TABLE 4).

The study done in lumber spinal stenosis in 2014 the ODI at 1 month and 24 months after operation were better than that of preoperative in two group respectively $(\mathrm{P}<0.05)$ and also the improvement of ODI in PTED group was better than that of the traditional surgery group $(\mathrm{P}<0.05)$.

Visual Analog Score (VAS) of leg and back pain from 7.5 to 1.7 and from 6.0 to 2.3. Our study also shows no different than other studies, the VAS of leg and back from $6.27+1.213$ to $1.40+-1.688$ and $4.79+1.162$ to $1.30+1.436$ with $\mathrm{p}=0.000, \mathrm{p}=0.00$ respectively before and after operation. VAS leg and Back with, $p=0.193$, $\mathrm{p}=0.897$ respectively showing no significant difference between LDH and LSS after surgery (GRAPH 1 and 2). In elderly patients the improvement of incision VAS in PTED group was better than that in the traditional surgery group $(\mathrm{P}<0.05)[39]$.

\begin{tabular}{|c|c|c|c|c|}
\hline \multicolumn{1}{|c|}{ Table 1. MacNab criteria. } & Percent & Valid Percent & Cumulative Percent \\
\hline E (Excellent) & Frequency & 40.3 & 40.3 & 40.3 \\
\hline G (Good) & 27 & 35.8 & 35.8 & 76.1 \\
\hline F (Fair) & 24 & 14.9 & 14.9 & 91.0 \\
\hline P (Poor) & 10 & 9.0 & 9.0 & 100.0 \\
\hline Total & 6 & 100.0 & 100.0 & \\
\hline
\end{tabular}

Table 2. MacNab score of patients in LDH and stenosis group.

\begin{tabular}{|c|c|c|c|c|}
\hline Group & E (Excellent) & G (Good) & F (Fair) & P (Poor) \\
\hline LDH & 22 & 13 & 7 & 3 \\
\hline Stenosis & 5 & 11 & 3 & 3 \\
\hline Total & 27 & 24 & 10 & 6 \\
\hline
\end{tabular}


Table 3. ODI of patients in LDH and stenosis

\begin{tabular}{|c|c|c|c|}
\hline & LDH & Stenosis & P value \\
\hline ODI & $-36.7276+19.16158$ & $-35.8850+17.70137$ & $\mathrm{p}=0.863$ \\
\hline
\end{tabular}

\begin{tabular}{|c|c|c|c|}
\hline \multicolumn{1}{|c|}{ Table 4. Pre and Postoperative ODI, VAS leg and VAS back. } \\
\hline & PRE OP & POST OP & P value \\
\hline ODI & $52.8022+11.98299$ & $16.3513+12.97398$ & $\mathrm{p}=0.000$ \\
\hline VAS LEG & $6.27+1.213$ & $1.40+1.688$ & $\mathrm{p}=0.000$ \\
\hline VAS BACK & $4.79+1.162$ & $1.30+1.436$ & $\mathrm{p}=0.000$ \\
\hline
\end{tabular}

VAS LEG

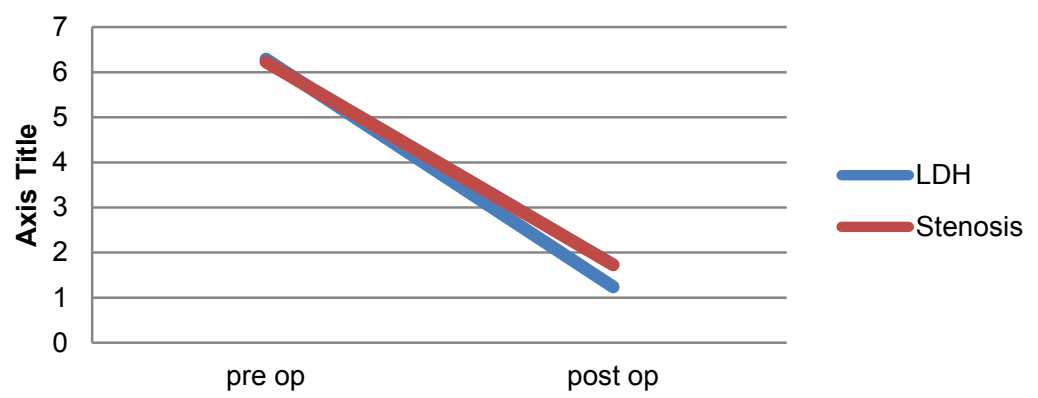

Graph 1. Comparison of pre and postoperative VAS of leg in LDH and Stenosis0.

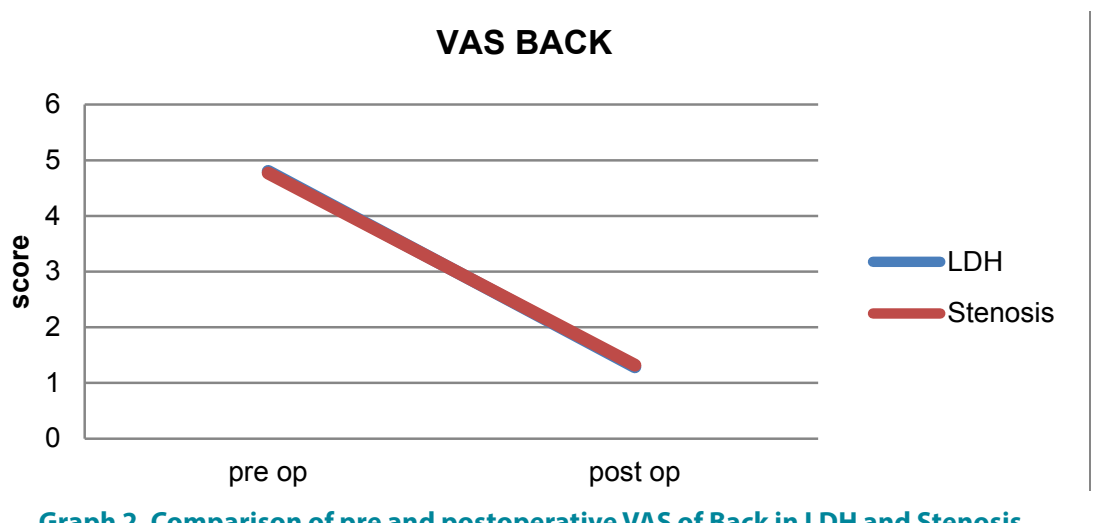

However, there may be a lot of effectiveness of the surgical treatment; there is always small risk of having complications [40]. Study also shows $1-2 \%$ case of Dural tear and 1-2\% case of nerve injury [41]. In our study no significant complication like Dural tear, dysesthesia and nerve root injuries were seen. Recently other studies show 3-6.6 rate of reherniation $[23,31,37,38,42]$. There was $10.5 \%$ out of which $3 \%$ (2 cases) of incomplete removal of content and $7.5 \%$ (5 cases) reherniation. 4 cases (8.9\%) of Reherniation was seen in $\mathrm{LDH}$ out of 45 cases and 1 case $(4.5 \%)$ of reherniation along with 2 cases $(9.1 \%)$ of incomplete removal of content was seen in LSS out of 22 cases. 5 patients of reherniation underwent further fusion surgery after few year. And incomplete removal of contents was again undergone revised PTES after 1 month of first surgery. Average hospital stay was $4.79+2.711$ days, which is similar to other studies. Mean duration of surgery was $87.31+24.746$ minutes.

\section{Conclusion}

The indications for Transforaminal Endoscopic treatment are the same as classical discectomy. Now due to advancement of instrumentation and skill of surgeon indication has broadened. In recent years, the clinical outcome of PTELS has increased with minimal complication due to advancement of instruments and skill of surgeons. Our study showed that there is no significant difference between the clinical outcome of Percutaneous Transforaminal Endoscopic Lumbar Surgery (PTELS) between Lumber disc herniation and 
Lumber spine stenosis of patient above 60 years age. Now PTELS can be alternative technique in spine surgery with satisfactory outcome, short hospital stay and no need of general anesthesia for elder patient who cannot usually tolerate general anesthesia. Therefore, the old aged patients with Low back pain can also have the significant benefits from the PTELS surgery. Though different studies show satisfactory clinical outcome further studies should be done. 


\section{REFERENCES}

Mäntyselkä P, Kumpusalo E, Ahonen $\mathrm{R}$, et al. Pain as a reason to visit the doctor: a study in Finnish primary health care. Pain 89(2), 175-180 (2001).

Hart LG, Deyo RA, Cherkin DC. Physician office visits for low back pain: frequency, clinical evaluation, and treatment patterns from a US national survey. Spine 20(1), 11-19 (1995).

Andersson GB. Epidemiological features of chronic low-back pain. The Lancet 354(9178), 581-585 (1999).

Panjabi MM. A hypothesis of chronic back pain: ligament subfailure injuries lead to muscle control dysfunction. Eur. Spine J. 15(5), 668-676 (2006).

Nachemson AL. Newest Knowledge of Low Back Pain A Critical Look. Clin. Orthop. Relat. Res. 279, 8-20 (1992).

Dewing CB, Provencher MT, Riffenburgh RH, Kerr S, Manos RE. The outcomes of lumbar microdiscectomy in a young, active population: correlation by herniation type and level. Spine 33(1), 3338 (2008).

Hoy D, Brooks P, Blyth F, Buchbinder R. The epidemiology of low back pain. Best Pract. Res. Clin .Rheumatol. 24(6), 769-781 (2010).

Parthan A, Evans CJ, Le K. Chronic low back pain: epidemiology, economic burden and patient-reported outcomes in the USA. Expert Rev. Pharmacoecon. Outcomes Res. 6(3), 359-369 (2006).

Pérennou, Dominique MD, Marcelli, et al. Epidemiologic Aspects in a LowBack Pain Population. Spine, 19(2), 123128 (1994).

Svensson HO, Andersson GB. The Relationship of Low-Back Pain, Work History, Work Environment, and Stress: A Retrospective Cross-Sectional Study of 38-to 64-Year-Old Women. Spine 14(5), 517-522 (1989).

Svensson HO, Andersson GB. Lowback pain in 40-to 47-year-old men: work history and work environment factors. Spine 8(3), 272-276 (1983).

Hartvigsen JK, Christensen,
Frederiksen H. Back pain remains a common symptom in old age. A population-based study of 4486 Danish twins aged 70-102. Eur. Spine J. 12(5), 528-534 (2003).

Waddell G. Volvo Award in Clinical Sciences: a new clinical model for the treatment of low-back pain. Spine 12(7), 632-644 (1987).

Smith WD, Dakwar E, Le TV, et al. Minimally invasive surgery for traumatic spinal pathologies: a mini-open, lateral approach in the thoracic and lumbar spine. Spine 35(26S), S338-346 (2010).

Sinkemani A, Hong X, Gao ZX, et al. Outcomes of microendoscopic discectomy and percutaneous transforaminal endoscopic discectomy for the treatment of lumbar disc herniation: a comparative retrospective study. Asian Spine J. 9(6), 833-840 (2015).

Postacchini F, Postacchini R. Operative Management of Lumbar Disc Herniation. In: Alexandre A., Masini M., Menchetti P. (eds) Advances in Minimally Invasive Surgery and Therapy for Spine and Nerves. Acta Neurochirurgica Supplementum, vol 108. Springer, Vienna, pp 17-21 (2011).

Birkenmaier C, Komp M, Leu HF, Wegener B. The current state of endoscopic disc surgery: review of controlled studies comparing full-endoscopic procedures for disc herniations to standard procedures. Pain Physician 16(4), 335-344 (2013).

Hirachan MP, Gao ZX, Lin Y, Singh R. Clinical Outcome of Percutaneous Endoscopic Lumbar Surgery (PELS) in Treatment of Lumbar Disc Herniation. Open J. Orthop. 7(04), 99 (2017).

Inui Y, Doita M, Ouchi K. Clinical and radiologic features of lumbar spinal stenosis and disc herniation with neuropathic bladder. Spine 29(8), 869873 (2004).

Justin SS, Alfred TO, Stephen S, Richard FG. Clinical outcomes after microendoscopic discectomy for recurrent lumbar disc herniation. J. Spinal Disorders Techniques 23(1), 30-34 (2010).

Carlsson AM. Assessment of chronic pain. I. Aspects of the reliability and validity of the visual analogue scale. Pain 16(1), 87-101 (1983).

Liao Z, Chen W, Wang $\mathrm{CH}$. Transforaminal percutaneous endoscopic surgery for far lateral lumbar intervertebral disk herniation. Orthopedics 37(8), e717-e727 (2014).

Ahn SS, Kim SH, Kim DW, Lee BH. Comparison of outcomes of percutaneous endoscopic lumbar discectomy and open lumbar microdiscectomy for young adults: a retrospective matched cohort study. World Neurosurg. 86, 250-258 (2016).

Knight MT. Endoscopically determined pain sources in the lumbar spine, in Lasers in the Musculoskeletal System. 2001, Springer. p. 267-273.

Mayer HM, Brock M. Percutaneous endoscopic discectomy: surgical technique and preliminary results compared to microsurgical discectomy. J. Neurosurg. 78(2), 216-225 (1993).

Kambin P, Gellman H. Percutaneous Lateral Discectomy of the Lumbar Spine A Preliminary Report. Clin. Orthop. Relat. Res. 174, 127-132 (1983).

Hijikata S. Percutaneous Nucleotomy: A New Concept Technique and 12 Years' Experience. Clin. Orthop. Relat. Res. 238, p. 9-23 (1989).

Forst R, Hausmann B. Nucleoscopy-a new examination technique. Arch. Orthop. Trauma Surg. 101(3), p. 219-221 (1983).

Yeung AT, Tsou PM. Posterolateral endoscopic excision for lumbar disc herniation: surgical technique, outcome, and complications in 307 consecutive cases. Spine. 27(7), p. 722-731 (2002).

Hoogland T, Schube DM, Miklit B, Ramirez A. Transforaminal posterolateral endoscopic discectomy with or without the combination of a low-dose chymopapain: a prospective randomized study in 280 consecutive cases. Spine. 31(24), p. E890-E897 (2006).

Ruetten S, Komp M, Merk H, Godolias G. Full-endoscopic interlaminar and transforaminal lumbar discectomy versus conventional microsurgical technique: a prospective, randomized, controlled study. Spine. 33(9), p. 931-939 (2008). 
Hoogland T, Miklit B, BrekelDijkstra K, Schubert M. Endoscopic transforaminal discectomy for recurrent lumbar disc herniation: a prospective, cohort evaluation of 262 consecutive cases. Spine. 33(9), p. 973-978 (2008).

Choy DS. Percutaneous laser disc decompression (PLDD): twelve years' experience with 752 procedures in 518 patients. J. Clin. Laser Med. Surg. 16(6), p. 325-331 (1998).

Burke SM, Safain MG, Kryzanski J, Riesenburger RI. Nerve root anomalies: implications for transforaminal lumbar interbody fusion surgery and a review of the Neidre and Macnab classification system. Neurosurg. Focus. 35(2), p. E9 (2013).

Tzaan W. Transforaminal percutaneous endoscopic lumbar discectomy. Chang Gung Med. J. 30(3), p. 226 (2007).
Lee JS, Kim HS, Jang JS, Jang IT. Structural Preservation Percutaneous Endoscopic Lumbar Interlaminar Discectomy for L5-S1 Herniated Nucleus Pulposus. BioMed Research International, Article ID 6250247 (2016).

Ahn Y, Lee SH, Park WM, et al. Percutaneous endoscopic lumbar discectomy for recurrent disc herniation: surgical technique, outcome, and prognostic factors of 43 consecutive cases. Spine. 29(16), p. E326-E332 (2004).

Li ZZ, Hou SX, Shang WS, Zheng C, Zhao HL. Percutaneous lumbar foraminoplasty and percutaneous endoscopic lumbar decompression for lateral recess stenosis through transforaminal approach: Technique notes and 2 years follow-up. Clin. Neurol. Neurosurg. 143, p. 90-94 (2016).

$\mathrm{Hu}$ DX, Zheng DX, Zhu DX, Ying DX, Wang DX. Percutaneous intervertebral foramina endoscopic lumbar discectomy decompression for elder patients with lumbar spinal stenosis syndrome. Zhongguo Gu Shang 27(3), p. 194-198 (2014).

Pan L, Zhang P, Yin Q. Comparison of tissue damages caused by endoscopic lumbar discectomy and traditional lumbar discectomy: a randomised controlled trial. Int. J. Surg. 12(5), p. 534-537 (2014).

Wang K, Hong X, Zhou BY, et. al. Evaluation of transforaminal endoscopic lumbar discectomy in the treatment of lumbar disc herniation. Int. Orthop. 39(8), p. 1599-1604 (2015).

Gadjradj PS, van Tulder MW, Dirven CM, Peul WC, Harhangi BS. Clinical outcomes after percutaneous transforaminal endoscopic discectomy for lumbar disc herniation: a prospective case series. Neurosurg. Focus. 40(2), E3 (2016).

This special issue on Current Trends in Clinical Research was edited by Dr. Mohamed Elsayed. 